J. Lake Sci. (湖泊科学), 2005, 17(2): 176-182

ISSN 1003 -5427; E-mail: jlakes@ niglas. ac.cn

Copyright 2005 by Journal of Lake Sciences

\title{
草型湖泊和藻型湖泊中大型底栖动物群落结构的比较”
}

间云君 ${ }^{1,2}$, 李晓宇 ${ }^{1}$, 梁彦龄 ${ }^{2}$

( 1 : 华中科技大学生命科学与技术学院,武汉 430074)

(2: 中国科学院水生生物研究所,武汉 430072)

摘要: 系统地比较了草型湖泊扁担塘与藻型湖泊后湖大型底栖动物的群落结构. 结果表明, 草型湖泊扁担塘的物种种 类、物种多样性、密度和生物量均较藻型湖泊后湖的为高. 两湖在物种组成和功能摄食群上存在极大差异, 相似性系数为 0.3 左右, 草型湖泊的大型底栖动物主要以刮食者数量为多, 而藻型湖泊主要以收集者的为多. 这说明水生高等植物对于 维持湖泊生态系统中大型底栖动物多样性方面起着非常重要的作用, 也是维持湖泊生态系统结构复杂性、空间异质性和 功能稳定性的重要因素.

关键词: 大型底栖动物; 群落结构; 生物多样性; $K$-优势曲线

\section{A Comparative Study on Community Structure of Macrozoobenthos Between Macrophtic and Algal Lakes}

\author{
YAN Yunjun ${ }^{1,2}$ \& Li Xiaoyu \& LIANG Yanling ${ }^{2}$ \\ ( 1 : School of Life Science and Technology, Huazhong University of Science and Technology, Wuhan 430074, P. R. China) \\ (2:Institute of Hydrobiology, Chinese Academy of Science, Wuhan 430072, P. R. China)
}

\begin{abstract}
A systematically comparative study on community structure of macrozoobenthos betweena a typical macrophytic lake, Lake Biandantang and a typical algal lake, Lake Houhu, was carried out from April, 1996 to March, 1997. The results showed that the species number, biodiversity, abundance and biomass of macrozoobenthos community in Lake Biandantang were much higher than those of macrozoobenthos in Lake Houhu; species composition and functional feeding groups were also prominently different, the similarity coefficient of macrozoobenthos communities in the two Lakes was about 0.30; in Biandantang Lake, scrapers were dominant in abundance and biomass, while in Houhu Lake, direct - collectors were dominant in number. These all probably were due to the presentation of higher aquatic macrophytes, especially those submerged ones, which provide various habitats and niches for more species, at the same time, keep the aquatic ecosystem complex, spatially heterogeneous and functionally stable.
\end{abstract}

Keywords : Macrozoobenthos, community structure, biodiversity, $K$-dominance curve

草型湖泊和藻型湖泊是湖泊演替过程中的不同阶段, 目前广泛存在于世界各国. 随着工农业的快速发 展, 城市人口的增多, 正促使这两种类型湖泊的转化速率日益加快, 这种情况在中国尤其明显. 为了遏制草 型湖泊水体富营养化和藻型化, 世界各国纷纷加快对这两种类型湖泊结构和功能的研究, 以期为将来治理 湖泊污染提供有效的解决方法 ${ }^{[1]}$. 以下报道的是我们在 1996 年至 1997 年间对草型湖泊扁担塘与藻型湖泊 后湖中大型底栖动物结构的比较研究结果.

\section{1 材料与方法}

\section{1 水体及样点设置}

选择浅水湖泊扁担塘和后湖为研究地点, 其中扁担塘为草型湖泊, 后湖为藻型湖泊. 两者采用了不同的 渔业模式,前者以生产河蟹、鳜鱼等名优水产品为主, 后者则以养殖鲢、鳙为主.

扁担塘: 为保安湖一子湖 (30015' N, 114043' E), 位于湖北省大冶市西北 $27 \mathrm{~km}$ 处, 保安湖的北部, 面积

* 国家自然科学基金(30270278,39600019 和 39430101) 资助. $2004-11-25$ 收稿;2005 - $02-04$ 收修改稿. 间云君, 男,1969 年生,博士, 教授; Email: yanyunjun@ Tom. com. 
$3.33 \mathrm{~km}^{2}$. 平均水深 $1.90 \mathrm{~m}$, 最大水深 $4.00 \mathrm{~m}$. 全湖几乎都有水草分布, 沿岸区域生长藕等挺水植物以及苦 草等沉水植物, 湖心生长聚草、金鱼藻等. 根据水草分布特点, 全湖共设 8 个采样点, 其中 S1、S6 点设在藕 区, S2、S3、S4 及 S5 点位于湖心断面上的聚草区, S7 点为苦草区, S8 点为无草区 (图 1a). 扁担塘水环境的理 化特性参数包括: 水深、透明度、水温、电导率、溶氧、 $\mathrm{pH}$ 值、总氮和总磷等, 由 “东湖生态站” 提供 (表 1 ).

表 1 扁担塘水环境的理化特性的周年动态

Tab. 1 Physical and chemical characteristics of lake water of Lake Biandantang

\begin{tabular}{lrrrrrrrrrrrr}
\hline 年. 月. 日 & 1996. & 1996. & 1996. & 1996. & 1996. & 1996. & 1996. & 1996. & 1996. & 1997. & 1997. & 1997. \\
& 4.26 & 5.19 & 6.18 & 7.27 & 8.21 & 9.20 & 10.16 & 11.18 & 12.24 & 1.22 & 2.27 & 3.28 \\
\hline 水深 $(\mathrm{m})$ & 1.39 & 1.35 & 1.33 & 2.06 & 2.79 & 2.1 & 2.18 & 2.36 & 1.54 & 1.46 & 1.38 & 1.1 \\
透明度 $(\mathrm{m})$ & 1.18 & 1.61 & 1.80 & 1.70 & 1.65 & 1.74 & 1.47 & 1.79 & 1.40 & 1.19 & 0.98 & 0.86 \\
水温 $\left({ }^{\circ} \mathrm{C}\right)$ & 22.81 & 22.86 & 30.45 & 30.02 & 30.59 & 26.00 & 20.83 & 11.09 & 10.04 & 7.18 & 13.3 & 15.4 \\
电导率 $(\mu \mathrm{S} / \mathrm{cm})$ & 250.75 & 246 & 241.4 & 328.5 & 205.9 & 204.9 & 190.7 & 166.1 & 172.1 & 190 & 310 & 315.5 \\
溶氧 $(\mathrm{mg} / \mathrm{L})$ & 10.99 & 11.26 & 7.25 & 8.15 & 9.34 & 9.78 & 13.32 & 12.05 & 8.55 & 13.48 & 11.8 & 10.82 \\
$\mathrm{pH}$ & 8.75 & 8.95 & 9.48 & 7.62 & 8.78 & 9.11 & 10.09 & 10.11 & 8.94 & 9.45 & 10.41 & 9.06 \\
$\mathrm{TN}(\mathrm{mg} / \mathrm{L})$ & 0.8014 & 1.202 & 0.608 & 0.715 & 1.53 & 0.65 & 0.7 & 0.75 & 2.283 & 3.815 & 2.58 & 4.335 \\
$\mathrm{TP}(\mathrm{mg} / \mathrm{L})$ & 0.059 & 0.089 & 0.060 & 0.032 & 0.056 & 0.050 & 0.044 & 0.040 & 0.054 & 0.013 & 0.020 & 0.028 \\
\hline
\end{tabular}

后湖: 为武汉东湖一子湖 (30033' N, 114023 ' E), 位于武汉市武昌区东北, 东湖的东南部, 面积 $3.33 \mathrm{~km}^{2}$. 平均水深 $2.21 \mathrm{~m}$, 最大水深为 $4.25 \mathrm{~m}$. 除沿岸区域生长藕等挺水植物以及苦草等沉水植物外, 湖 底绝大部分为裸露泥底. 根据湖泊的环境及生态特征, 在湖中心设置四个采样点: St 1 、St2、St3、St4 (图 1b). 后湖水环境的理化特性参数亦包括: 水深、透明度、水温、电导率、溶氧、 $\mathrm{pH}$ 值、总氮和总磷等, 由中国科学院 东湖生态站提供(表 2).

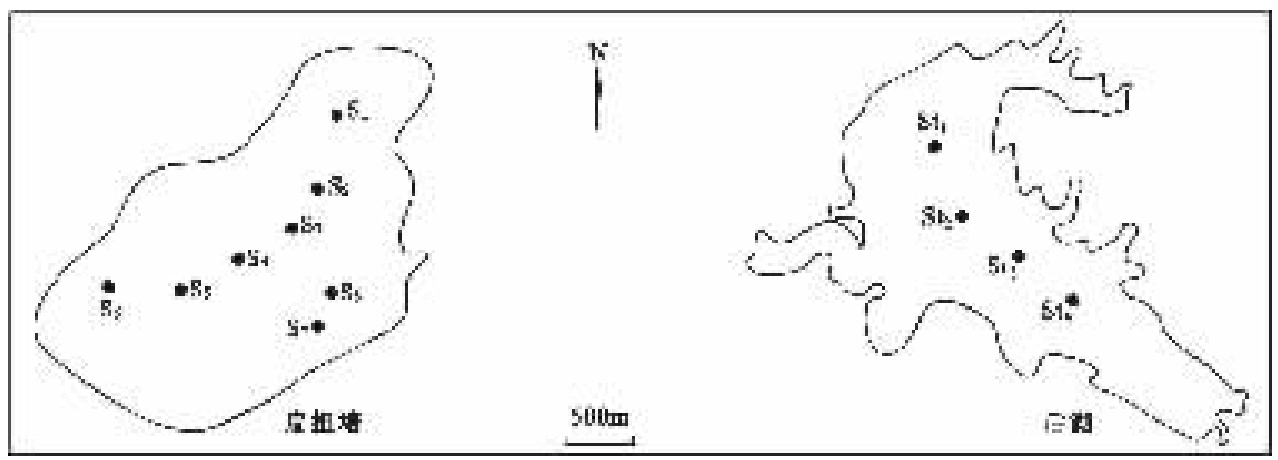

图 1 扁担塘及后湖的采样点

Fig. 1 Distribution of sampling sites of Lake Biandantang and Lake Houhu

\section{2 采样时间}

扁担塘:一般在每月 20 日左右,为期一年,即 1996 年 4 月至 1997 年 3 月.后湖:一般在每月 15 日左右, 为期一年, 即 1996 年 4 月至 1997 年 3 月.

\section{3 采样方法}

定性采样: 除使用 $1 / 16 \mathrm{~m}^{2}$ 改进 Petersen 采泥器每月每样点采集一次外, 还在湖边采集了大量底栖动物 标本, 用于定性鉴定. 为了鉴定摇蚊种类, 收集了数百只摇蚊成虫标本. 标本用 $10 \%$ 的福尔马林液固定 保存.

定量采样:使用 $1 / 16 \mathrm{~m}^{2}$ 改良彼得生式采泥器, 每月每点一次, 泥样经 60 目 $(167 \mu \mathrm{m})$ 的铜耖或纱网篮 洗后, 置于解剖盘中分检, 标本用 $10 \%$ 的福尔马林固定. 
表 2 后湖水环境的理化特性的周年动态

Tab. 2 Physical and chemical characteristics of lake water of Lake Houhu

\begin{tabular}{lrrrrrrrrrrrr}
\hline \multicolumn{1}{c}{ 年.月. } & 1996. & 1996. & 1996. & 1996. & 1996. & 1996. & 1996. & 1996. & 1996. & 1997. & 1997. & 1997. \\
& 4.29 & 5.16 & 6.13 & 7.15 & 8.15 & 9.16 & 10.14 & 11.14 & 12.10 & 1.17 & 2.18 & 3.17 \\
\hline 水深 $(\mathrm{m})$ & 2.69 & 2.92 & 3.075 & 3.55 & 2.7 & 2.7 & 2.63 & 2.73 & 2.63 & 2.28 & 1.93 & 2.75 \\
透明度 $(\mathrm{m})$ & 1.45 & 0.845 & 0.875 & 1.03 & 1.075 & 0.87 & 1 & 0.65 & 0.7 & 0.17 & 0.58 & 0.48 \\
温度 $\left({ }^{\circ} \mathrm{C}\right)$ & 21.94 & 20.63 & 26.23 & 26.62 & 31.27 & 27.31 & 20.64 & 12.16 & 8.65 & 6.16 & 9.22 & 11.69 \\
电导率 $(\mu \mathrm{S} / \mathrm{cm})$ & 302.0 & 308.5 & 280.7 & 218.0 & 189.0 & 208.5 & 199.0 & 170.3 & 165.5 & 165.5 & 310.0 & 300.5 \\
$\mathrm{DO}(\mathrm{mg} / \mathrm{L})$ & 9.59 & 10.54 & 10.15 & 6.78 & 8.56 & 7.84 & 11.34 & 12.78 & 10.13 & 5.85 & 12.24 & 10.5 \\
$\mathrm{pH}$ & 7.97 & 7.88 & 8.1 & 7.7 & 8.19 & 8.87 & 8.65 & 9.49 & 8.85 & 10.66 & 10.45 & 8.94 \\
$\mathrm{TN}(\mathrm{mg} / \mathrm{L})$ & 2.87 & 2.465 & 0.824 & 0.82 & 1.194 & 2.076 & 1.594 & 1.12 & 1.03 & 2.818 & 3.188 & 2.45 \\
$\mathrm{TP}(\mathrm{mg} / \mathrm{L})$ & 0.049 & 0.101 & 0.066 & 0.006 & 0.039 & 0.072 & 0.054 & 0.045 & 0.116 & 0.0302 & 0.041 & 0.056 \\
\hline
\end{tabular}

\section{4 数据分析}

采用 Jørgensen 指数、Jaccard 相似性指数比较扁担塘和后湖大型底栖动物群落结构的差异; 物种多样性 用 Margalef、Simpson、Shannon-Wiener 多样性指数评估; 用 K-优势曲线的方法比较扁担塘和后湖大型底栖动 物的物种多样性. 采用双因素方差 (ANOVA) 检验各采样点之间和扁担塘与后湖之间的大型底栖动物物种 数及密度的差异.

\section{2. 结果}

\section{1 两个湖泊底栖动物群落构成}

经鉴定, 扁担塘共发现大型底栖动物 88 种, 其中寡毛类 29 种, 昆虫 32 种, 软体动物 17 种, 其它动物 10 种; 后湖有 44 种, 其中寡毛类 15 种, 昆虫 15 种, 软体动物 12 种, 其它动物 2 种 (表 3 ). 两者存在显著差异 $(P<0.05)$. 两湖的主要类群寡毛类、软体动物和水生昆虫的种类差异也极为显著 $(P<0.01)$, 扁担塘的种 类更多为周丛生物, 以水草为生存生境, 取食和繁殖.

\section{2 两湖大型底栖动物群落相似性}

从上述名录可知, 后湖、扁担塘大型底栖动物群落共有物种为: 普通仙女虫、简明仙女虫、参差仙女虫、 豹行仙女虫、多突癞皮虫、尖头杆吻虫、印西头鳃虫指鳃尾盘虫、叉形管盘虫、苏氏尾鳃蚓、霍甫水丝蚓、泥氏 癞颤蚓、维窦夫盘丝蚓、花纹前突摇蚊、大红德永摇蚊、羽摇蚊、铜锈环棱螺、梨形环棱螺、长角涵螺、纹沼螺、 方格短沟蜷、狭夢卜螺、椭圆萝卜螺、圆顶珠蚌、湖球蚬.一共 25 种.

Jørgensen 指数: $S=2 c /(a+b)$ (式中, $S$ 相似性指数; $c$ : 两个群落共有的种数; $a$ : 扁担塘大型底栖动 物群落中的物种数; $b$ 后湖大型底栖动物群落中的物种数) 计算扁担塘、后湖大型底栖动物群落的相似性系 数为: $\mathrm{S}=25 \times 2 /(44+88)=0.38$.

Jaccard 相似性指数: $S_{j}=c /(a+b-c)=25 /(44+88-25)=0.23$.

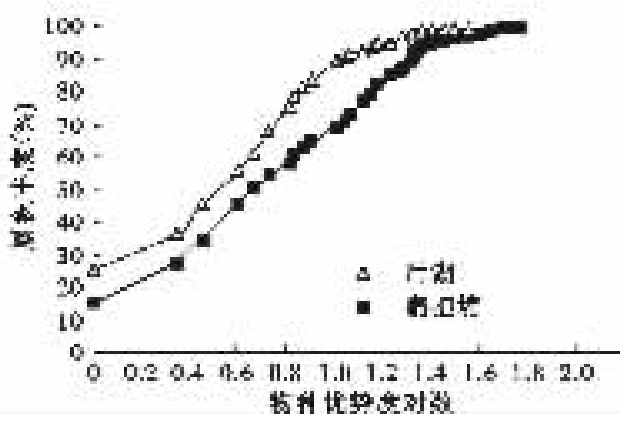

图 2 两湖大型底栖动物的 $K$ - 优势曲线

Fig. $2 K$-dominant curves of macrozoobenthos in

Lake Biandantang and Lake Houhu
两种相似性指数均说明扁担塘与后湖底栖动物群落结 构存在较大差异.

\section{3 两湖大型底栖动物生物多样性}

物种多样性可用 $K-$ 优势曲线比较, 它综合了物种多 样性的两个主要方面一一物种丰富度和均匀性, 通过对一 特定群落内的各个物种 (按优势度从大到小排列) 相应的 累计密度百分数作图, 群落的优势格局可以非常直观地表 现出来. 如果某一曲线所有点都位于另一曲线之下, 表明该 曲线所表示的群落物种多样性比另一曲线表示的群落高. 图 2 是扁担塘和后湖大型底栖动物群落的 $K-$ 优势曲线. 从 图 2 可以看出, 扁担塘大型底栖动物群落多样性要高于后湖 的. 同样的结论也可以从两湖物种多样性指数得出 (表 4). 
表 3 扁担塘、后湖大型底栖动物群落构成

Tab. 3 Composition of macrozoobenthos community in the two lakes

\begin{tabular}{|c|c|c|c|c|c|}
\hline 类 & 扁担塘 & 后湖 & 种 & 扁担塘 & 后湖 \\
\hline 寡毛类 Oligchaeta & & & 褐斑菱跗摇蚊 Clinotanypus sugiyamai & & + \\
\hline 透清毛腹虫 Chaetogaster diaphanus & + & & 花纹前突摇蚊 Procladius choreus & + & + \\
\hline 椎实毛腹虫 Ch. limnaei & + & & 粗腹摇蚊一种 Pelopia sp. 1 & + & \\
\hline 郎氏毛腹虫 $C h$. langi & + & & 刺铗粗腹摇蚊 $P$. punctipennis & + & \\
\hline 盘缠毛腹虫 Ch. diastrophus & + & & 粗腹摇蚊一种 Tanypus sp. 2 & & + \\
\hline 费氏拟仙女虫 Paranais frici & + & & 双线环足摇蚊 Cricotopus bicinctus & + & \\
\hline 约氏斯必卡虫 Specaria josinae & & + & 流放环足摇蚊 C. exilis & + & \\
\hline 普通仙女虫 Nais communis & + & + & 三横带真环足摇蚊 Eucricotopus trifasciatus & + & \\
\hline 简明仙女虫 $N$. simplex & + & + & 直突摇蚊一种 Orthocladius sp. & + & \\
\hline 参差仙女虫 $N$. variabilis & + & + & 长跗摇蚊一种 Tanytarsus sp. + & & \\
\hline 多突癞皮虫 Slavina appendiculata & + & + & 大红德永摇蚊 Tokunagayusurika akamushi & + & + \\
\hline 有栉异仙女虫 $A$. pectinata & + & & 异拟长跗摇蚊 Paratanytarsus dissimilis & + & \\
\hline 巴拉圭异仙女虫 A. paraguayensis & + & & 项圈五脉摇蚊 Pentaneura monilis & + & \\
\hline 豹行仙女虫 $N$. pardalis & + & + & 伸展摇蚊 C. tenans & + & \\
\hline 尖头杆吻虫 Stylaria fossularis & + & + & 羽摇蚊 C. plumosus & + & + \\
\hline 印西头鳃虫 Branchiodrilis hottensis & + & + & 细长摇蚊 Chironomus attenuatus & + & \\
\hline 指鳃尾盘虫 Dero digitata & + & + & 指突隐摇蚊 Cryptochironomus digitatus & + & \\
\hline 钝尾盘虫 D. obtusa & + & - & 翠绿隐摇蚊 $C$. viridulus & + & \\
\hline 叉形管盘虫 Aulophorus furcatus & + & + & 软铗隐摇蚊 Cryptochironomus tener & & \\
\hline 长毛吻盲虫 Pristina longiseta & + & - & 摇蚊一种 Chironomus sp. & & \\
\hline 尖头小吻盲虫 Pristinella. accuminata & + & - & 侧叶雕翅摇蚊 Glyptochironomus lobiferus & + & \\
\hline 奥氏小吻盲虫 $P$. osborni & + & - & 沼摇蚊 Limnochironomus nervosus & + & \\
\hline 等毛吻盲虫 Pristina aquiseta & - & + & 梯形多足摇蚊 Polypedilum scalaenum & + & \\
\hline 中华河蚓 Rhyacodrilus sinicus & - & + & 异腹鳃摇蚊 Einfeldia insolita & + & \\
\hline 苏氏尾鳃蚓 Branchiura sowerbyi & + & + & 摇蚊一种 Chironomus sp. & + & \\
\hline 霍甫水丝蚓 L. hoffmeisteri & + & + & 蠓科一种 Palpomyia sp. & + & \\
\hline 巨毛水丝蠫 L. grandisetosus & + & - & 幽蚊一种 Chaoborus sp & + & \\
\hline 多毛管水蚓 Aulodrilus pluriseta & + & - & 蟌一种 Caenagidae sp. & & + \\
\hline 皮氏管水蚓 A. pigueti & + & - & 瘦蟌一种 Ichnura sp. & + & \\
\hline 正颤蚓 Tubifex tubifex & + & - & 蟌一种 Cerion sp. & & + \\
\hline 尼式癞颤蚓 Spirosperma nikolskyi & + & + & 蜓一种 Aeschna $\mathrm{sp}$. & & + \\
\hline 维窦夫盘丝蚓 B. vejdovskyanum & + & + & 赤足 Sympetrum sp. & + & \\
\hline 软体动物 Mollusca & & & 黄蜻 Patala sp. & & + \\
\hline 铜锈环棱螺 Bellamya aeruginosa & + & + & 蜉蝣 Ephemera valgata & + & \\
\hline 梨形环棱螺 B. purificata & + & & 细蝣 Caenis macrura & + & \\
\hline 长角涵螺 Alocinma longicornis & + & + & 细蝣一种 Caenis sp. & & + \\
\hline 纹沼螺 Parafossarulus striatulus & + & + & 管石虫种 1 Ecnomus sp. 1 & + & \\
\hline 中华沼螺 $P$. sinensis & + & & 管石蛾种 2 Ecnomus sp. 2 & & + \\
\hline 大沼螺 P. eximius & + & & 长角石蟇 Setodes biwa & + & \\
\hline 榭豆螺 Bithynia misella & & + & 石蛾一种 Phryganea sp. & + & \\
\hline 方格短沟蜷 $S$. cancellata & + & + & 沼石蛾一种 Limnophilus sp. & + & \\
\hline 短沟蜷种 1 Semisulcospira sp. 1 & + & & 毛翅目一种 Neureclipsis sp. & + & \\
\hline
\end{tabular}




\begin{tabular}{|c|c|c|c|c|c|}
\hline 种 类 & 扁担塘 & 后湖 & 类 & 扁担塘 & 后湖 \\
\hline 短沟虫卷种 2 Semisulcospira sp. 2 & & + & 其他动物 Other animals & + & \\
\hline 狭萝卜螺 Radix lagotis & + & + & 武昌罗索线虫 Romanomermis wuchangensis & + & \\
\hline 椭圆萝卜螺 $R$. ovata & + & + & 沼索线虫 (种 1 ) Limnomermis sp. 1 & + & \\
\hline 圆扁螺 Hippeutissp. & + & & 沼索线虫 (种 2) Limnomermis sp. 2 & & + \\
\hline 凸旋螺 Gyraulus conexiusculus & & + & 扁蛭一种 Glossiphonia sp. 1 & + & \\
\hline 旋螺 Gyraulus sp. & & + & 扁蛭一种 Glossiphonia sp. 2 & & + \\
\hline 光滑狭口螺 Stenothyra glabra & + & & 拟扁蛭一种 Hemiclepsis sp. & + & \\
\hline 短褶矛蚌 Lanceolaria grayana & + & & 泽蛭一种 Helobdella sp. & + & \\
\hline 圆顶珠蛙 Unio douglasiae & + & + & 金线蛭一种 Whitmania sp. & + & \\
\hline 淡水壳菜 Limnoperna lacustris & & & 医蛭一种 Hirudo sp. & & + \\
\hline 湖球蚬 Sphaerium lacustre & + & + & 端足类 Amphipoda & + & \\
\hline 河蚬 Corbicula fluminea & + & & 等足类 Isopoda & + & \\
\hline $\begin{array}{l}\text { 水生昆虫 Insecta } \\
\text { 微刺菱跗摇蚊 C. }\end{array}$ & + & & 米虾一种 Caridina sp. & + & \\
\hline
\end{tabular}

表 4 扁担塘和后湖大型底栖动物群落多样性指数

Tab. 4 Biodiversities of macrozoobenthos in Lake Biandantang and Houhu

\begin{tabular}{ccc}
\hline 多样性指数 & 扁担塘 & 后湖 \\
\hline Shannon - Wiener 指数 & 2.89 & 2.18 \\
Margalef 指数 & 9.12 & 4.23 \\
Simpson 指数 & 7.64 & 4.52 \\
\hline
\end{tabular}

\section{4 功能摄食类群}

通常将水生无脊椎动物分为 4 类:撕食者、收集者(包括直接收集者和过滤收集者)、刮食者和捕食者. 近年对淡水生态系统中底栖动物的摄食类群有较多的研究, 以期更多了解底栖动物在群落食物网中的营养 地位及生态功能, 并可根据各类群的分布和比例划分水体区域, 以利于科学管理. 扁担塘和后湖的大型底栖 动物功能摄食群分布见表 5. 从表中可知, 扁担塘和后湖的大型底栖动物以收集者为多, 其密度也较多, 但 在扁担塘虽然刮食者种类不如收集者多, 但密度占据优势, 这与扁担塘密生着多种高等水生植物, 特别是沉 水植物使生态系统具有复杂的结构, 并为周丛藻类提供了附着基质, 为刮食者提供了充足生存空间和食物 有关. 后湖由于没有水草,适合直接收集者生活,因而占据数量优势.

表 5 扁担塘和后湖大型底栖动物的功能摄食类群

Tab. 5 The functional feeding groups in Lake Biandantang and Lake Houhu

\begin{tabular}{|c|c|c|c|c|}
\hline \multirow{2}{*}{ 功能摄食类群 } & \multicolumn{2}{|c|}{ 扁担塘 } & \multicolumn{2}{|c|}{ 后湖 } \\
\hline & 种类数量 & 密度 (ind. $/ \mathrm{m}^{2}$ ) & 种类数量 & 密度 (ind. $/ \mathrm{m}^{2}$ ) \\
\hline 收集者 & 57 & 234 & 21 & 262 \\
\hline 刮食者 & 13 & 305 & 9 & 69 \\
\hline 捕食者 & 11 & 178 & 7 & 214 \\
\hline 撕食者 & 5 & 123 & 1 & 20 \\
\hline
\end{tabular}

\section{5 密度和生物量}

扁担塘大型底栖动物的年均密度和生物量均比后湖的为高, 分别是 $840 \mathrm{ind} / \mathrm{m}^{2} 、 40.3 \mathrm{~g} / \mathrm{m}^{2}, 565 \mathrm{ind} /$ $\mathrm{m}^{2} 、 26.8 \mathrm{~g} / \mathrm{m}^{2}$. 在扁担塘, 摇蚊幼虫、软体动物和寡毛类占底栖动物总密度的绝大多数, 分别为 $41 \% 、 35 \%$ 和 $17 \%$; 与之相比, 后湖中摇蚊幼虫和寡毛类占总密度的大多数, 分别是 $73 \%$ 和 $21 \%$. 在生物量方面, 软体动 物在两湖中均占有绝对优势,在扁担塘和后湖分别为 $91 \%$ 和 $84 \%$. 

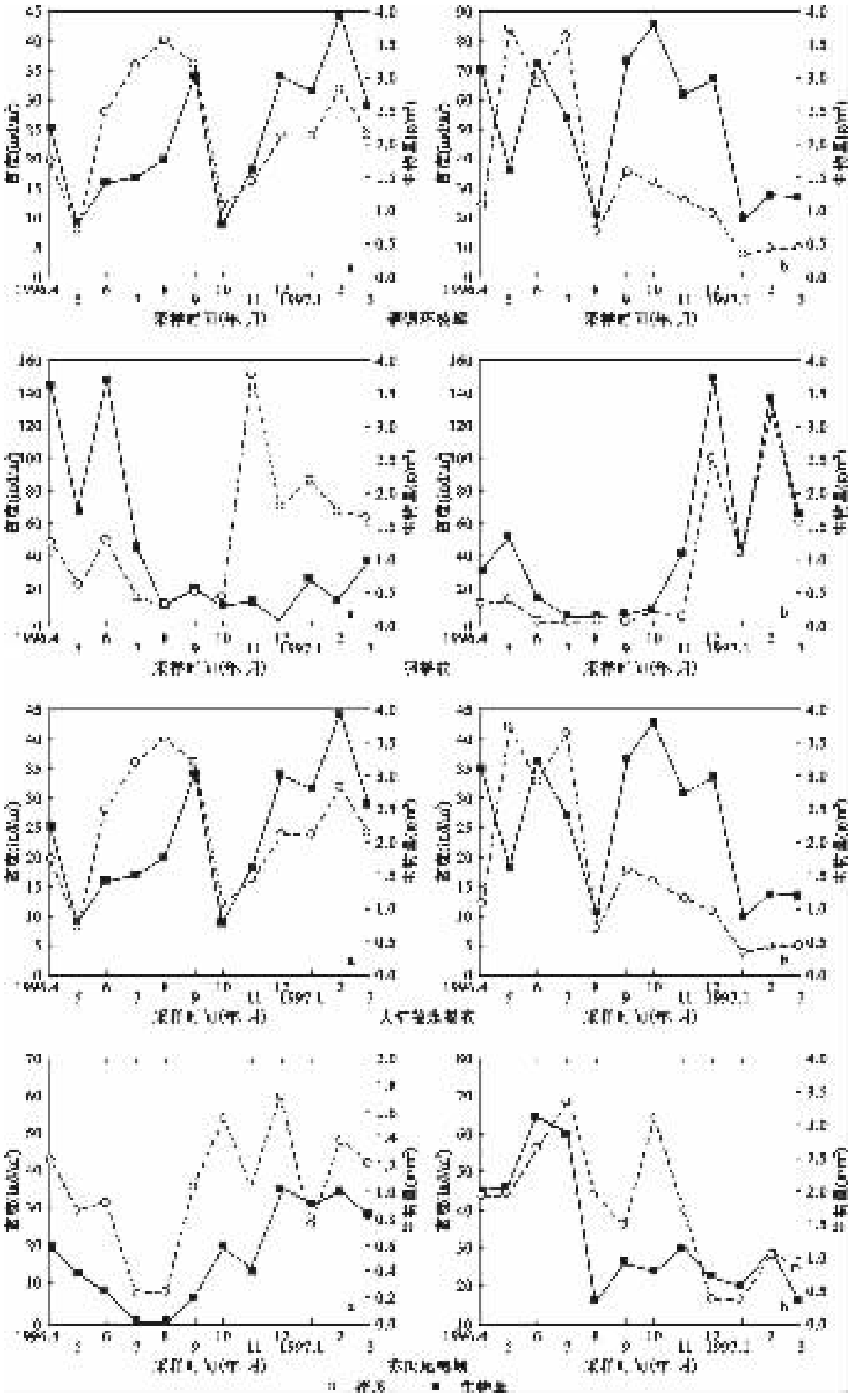

图 3 扁担塘、后湖大型底栖动物主要优势种密度 $\left(\mathrm{ind} / \mathrm{m}^{2}\right)$ 和生物量 $\left(\mathrm{g} / \mathrm{m}^{2}\right)$ 的周年动态 Fig. 3 Annual variations of densities $\left(\mathrm{ind} / \mathrm{m}^{2}\right)$ and biomass $\left(\mathrm{g} / \mathrm{m}^{2}\right)$ 


\section{6 优势种群的周年动态}

扁担塘的主要优势种为苏氏尾鳃蚓、大红德永摇蚊、羽摇蚊、铜锈环棱螺、长角涵螺、纹沼螺. 后湖的优 势种为苏氏尾鳃蚓、霍甫水丝蚓、大红德永摇蚊、羽摇蚊、铜锈环棱螺. 扁担塘和后湖的共有优势种现存量周 年动态如图 3. 扁担塘除有草型湖泊特有的刮食者长角涵螺、纹沼螺进入优势种行列, 其它优势种与后湖基 本相同, 后湖拥有藻型湖泊代表性优势种霍甫水丝蚓. 但从现存量周年动态看, 除大红德永摇蚊的变动趋势 相近外,其它相同各种间均存在一定差异,这可能与它们长期适应两种不同的生境有关.

\section{3 讨论}

从物种数、现存量和物种多样性看, 扁担塘具有比后湖更为丰富的大型底栖动物群落, 表明在淡水浅水 湖泊中水生植物特别是高等水生植物对维持大型底栖动物多样性方面起着极为重要的作用. 大量的水生植 物直接改变了湖泊生态系统的空间结构, 并增加了空间的异质性. 按照 $\operatorname{Simpson}^{[2]}$ 的空间异质性理论, 环境 的理化条件越复杂, 生物的区系也就越多样化. 高度空间异质性不仅为大型底栖动物提供了栖息、生活、摄 食和繁殖的场所, 也为底栖动物提供了躲避捕食者良好条件. 尽管扁担塘杂食性鱼类和专食性鱼类的捕食 压力较后湖的大 ${ }^{[3,4]}$, 但扁担塘水生植物提供的良好隐蔽条件, 使大型底栖动物仍然保持着较高的多度和 物种多样性.

除上述原因外, 食物的差异性也是一个重要的原因. 草型湖泊中水草也为多种藻类、细菌、真菌和原生 动物等低等生物类群提供了生活场所, 为大型底栖动物特别是刮食者提供了充足的食物来源 ${ }^{[5]}$. 相比, 藻 型湖泊没有这样的空间结构, 不可能提供多样性食物条件, 制约着物种多样性发展, 但促使某些适应种类发 展出数量较大的种群, 如后湖的霍甫水丝蚓.

类似的现象在其它类群中也存在,如扁担塘的自由线虫、鱼类也均比后湖的多样性和现存量高 ${ }^{[4]}$ (1).

\section{4 参考文献}

[1] 梁彦龄, 刘伙泉主编. 草型湖泊资源、环境与渔业生态学管理 (一). 北京:科学出版社, $1995: 245$.

[2] Simpson G G. Species density of North American recent mammals. Systematic Zoology, 1964, 13:57 - 73.

[3] 张堂林, 方榕乐, 崔亦波. 渔业发展阶段不同的五个水体鱼类多样性的比较. 水生生物学报, 1996,20(增刊) : 191 $-198$.

[4] 黄根田, 谢 平. 武汉东胡鱼类群落结构的变化及原因的分析. 水生生物学报, 1996,20(增刊):38-46.

[5] Engel S. The role and interaction of submerged macrophytes in a shallow Wisconsin lake. Journal of Freshwater Ecology, $1998,4: 329-341$. 